Pacific Journal of Mathematics

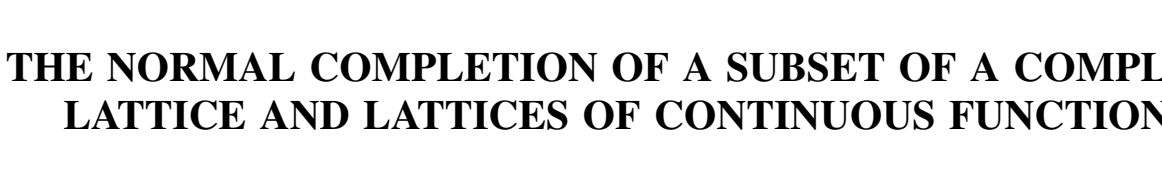




\title{
THE NORMAL COMPLETION OF A SUBSET OF A COMPLETE LATTICE AND LATTICES OF CONTINUOUS FUNCTIONS
}

\author{
ALFRED HORN
}

Introduction. The normal completion of a partially ordered set $C$ is a complete lattice $\widetilde{C}$ whose elements are subsets of $C$. However, in practice a partially ordered set $C$ is usually given as a subset of a complete lattice $B$, and it seems desirable to be able to describe directly a subset of $B$ which contains $C$ and which is isomorphic with $\tilde{C}$. Such a construction is given in $\S 1$ below. The idea of the construction is suggester by the method of formation of the normal completion itself, and is undoubtedly known. However, it does not seem to appear explicitly in the literature. In $\delta 2$ we treat the case where $C$ is the lattice of real-valued continuous functions on a topologic space $X$, and $B$ is the lattice of all real-valued functions on $X$. This leads to a simple proof of a result of Dilworth [2]. In $\$ 3$ we extend another result of Dilworth in proving that for any two topologic spaces, the lattices of regular open sets are isomorphic if and only if the lattices of normal lower semicontinuous functions are isomorphic.

Notations and definitions. If $S$ is a subset of a partially ordered set $P$, then $\sum_{P}(S)$ and $\Pi_{P}(S)$ will be used to denote the least upper bound and the greatest lower bound of $S$, respectively. If $S$ is empty, $\sum_{P}(S)$ is the least element of $P$, and $\prod_{P}(S)$ is the greatest element of $P$. A subset of $P$ will always be given the order induced by that of $P$. If $x \in P, S \subset P$, we shall write $x \geq S$ whenever $x \geq y$ for all $y$ in $S$. Two partially ordered sets $P_{1}$ and $P_{2}$ are said to be isomorphic, in symbols $P_{1} \cong P_{2}$, if there exists a function $f$ on $P_{1}$ onto $P_{2}$ such that

$$
x \leq y \leftrightarrow f(x) \leq f(y) .
$$

This implies that $f$ is one-to-one.

If $A$ is a subset of a topologic space, we use $d A$, and $C A$ for the interior and the closure of $A$. A set $S$ is called regular open if $S=d C S$, and regular closed if $S=\mathrm{Cd} S$. A zero-dimensional space is one whose open sets have a basis each member of which is both open and closed. A space shall be called

Received December 2, 1951.

Pacific J. Math. 3 (1953), 137-152 
extremally disconnected if every regular open set is closed, that is, if the closure of every open set is open. Contrary to the usual custom, we do not require a completely regular space to satisfy any of the separation axioms. If $A$ is a set, $k_{A}$ will stand for the characteristic function of $A$. If $f$ is a function, we use $E(f>\lambda)$ to denote $E_{x}(f(x)>\lambda)$.

1. The normal completion of a subset of a complete lattice. I.et $B$ be a complete lattice on which are defined a "closure operation" $x^{*}$ and a "dual closure operation" $x_{*}$ with the following properties:

i) $\quad x_{*} \leq x \leq x^{*}$,

ii) $\left(x_{*}\right)_{*}=x_{*},\left(x^{*}\right) *=x^{*}$,

iii) $x \leq y \longrightarrow x_{*} \leq y_{*}$ and $x^{*} \leq y^{*}$.

As a simple consequence, we have

iv) $\left(\left(\left(x_{*}\right) *\right)_{*}\right) *=\left(x_{*}\right) *$ and $\left(\left(\left(x^{*}\right)_{*}\right) *\right)_{*}=\left(x^{*}\right)_{*}$.

Throughout this section, $\sum(S)$ and $\Pi(S)$ will be used to denote $\sum_{B}(S)$ and $\prod_{B}(S)$. Let

$$
L=E_{x}\left(x=x_{*}\right) \text {, and } U=E_{x}\left(x=x^{*}\right) \text {. }
$$

A proof of the following theorem is given in $[1, \mathrm{p} .49]$.

THEOREM 1. L and $U$ are complete lattices in which

$$
\begin{array}{ll}
\sum_{L}(S)=\sum(S), & \Pi_{L}(S)=\Pi(S), \\
\sum_{U}(S)=\sum(S)^{*}, & \Pi_{U}(S)=\Pi(S) .
\end{array}
$$

Now let

$$
N L=E_{x}\left(x=\left(x^{*}\right)_{*}\right) \text {, and } N U=E_{x}\left(x=\left(x_{*}\right) *\right) \text {. }
$$

We note that $N L \subset L$ and $N U \subset U$, by ii $)$.

THEOREM 2. NL and NU are isomorphic complete lattices in which

$$
\begin{aligned}
& \sum_{N L}(S)=\left(\sum(S)^{*}\right)_{*}, \prod_{N L}(S)=\prod(S)_{*}, \\
& \sum_{N U}(S)=\sum(S)^{*}, \prod_{N U}(S)=\left(\prod_{\left.(S)_{*}\right)^{*} .}\right.
\end{aligned}
$$


Proof. It is easy to verify by i), ii), iii), and iv) that $\left(x^{*}\right)_{*}$ is a closure operation in $L$. By Theorem 1 it follows that $N L$ is a complete lattice in which

$$
\sum_{N L}(S)=\left(\sum_{L}(S)^{*}\right)_{*}=\left(\sum(S)^{*}\right)_{*}, \prod_{N L}(S)=\prod_{L}(S)=\prod(S)_{*} \text {. }
$$

For the other half of the proof, we define the dual closure operation $\left(x_{*}\right) *$ in $U$, and apply Theorem 1 again. The mapping $f(x)=x^{*}$ is easily seen to be an isomorphism of $N L$ onto $N U$ whose inverse is $f^{-1}(x)=x_{*}$.

As an example, if $B$ is the lattice of all subsets of a topologic space, and

$$
x_{*}=d x, x^{*}=\mathrm{C} x,
$$

then $L$ and $U$ are the lattices of open sets and closed sets, while $N L$ and $N U$ are the lattices of regular open sets and regular closed sets respectively.

The normal completion (completion by cuts) of a partially ordered set $C$ is a canonical embedding of $C$ in a complete lattice $\widetilde{C}$ which preserves all existing sums and products. See $[1$, p. 58] for details. Now $\tilde{C}$ may be constructed as follows. If $S \subset C$, let

$$
S^{\wedge}=E_{x}(x \in C, x \geq S) \text { and } \bar{S}=E_{x}\left(x \in C, x \leq S^{\wedge}\right) .
$$

Then $\bar{S}$ is a closure operation in the lattice of all subsets of $C$. The class

$$
\tilde{C}=E_{S}(S=\bar{S})
$$

is the normal completion of $C ; C$ is embedded in $\widetilde{C}$ by the mapping

$$
f(x)=\bar{S}_{x},
$$

where $S_{x}$ is the class whose only member is $x$.

Now suppose $C$ is an arbitrary subset of $B$. Our aim is to describe a subset of $B$ which is isomorphic with $\tilde{C}$. If $x \in B$, we define

$$
x_{0}=E_{y}(y \in C, y \leq x) \text { and } x^{0}=E_{y}(y \in C, y \geq x) \text {. }
$$

Now let

$$
x_{*}=\sum\left(x_{0}\right), x^{*}=\prod\left(x^{0}\right) .
$$

It is easily verified that $x^{*}$ and $x_{*}$ are closure and dual closure operations on $B$. To check property ii), we need only note that if $y \in C$, then 


$$
y \leq x_{*} \leftrightarrow y \leq x
$$

We also remark that

$$
x \in C \rightarrow x^{*}=x=x_{*} \text {. }
$$

We now consider the lattices $L, U, N L$, and $N U$ obtained from this particular definition of $x^{*}, x_{*}$. Oibviously $C \subset N L, C \subset N U$, and the following theorem shows that $N L$ and $N U$ are extensions of $C$.

THEOREM 3. If

$$
x=\sum_{C}(S), y=\prod_{C}(S)
$$

then

$$
x=\sum_{N L}(S)=\sum_{N U}(S) \text { and } y=\prod_{N L}(S)=\prod_{N U}(S) .
$$

'roof. First

$$
w \in C, w \geq \sum(S) \rightarrow w \in C, w \geq S \rightarrow w \geq \sum_{C}(S)=x .
$$

lience

$$
\sum(S)^{0} \geq x
$$

and therefore

$$
\sum(S)^{*} \geq x
$$

Also

$$
\sum(S)^{*} \leq x
$$

since $x \in C$ and $x \geq \sum(S)$. Thus

$$
x=\sum(S)^{*}=\sum_{N U}(S) .
$$

Also, since $x \in C$,

$$
x=x_{*}=\left(\sum(S)^{*}\right)_{*}=\sum_{N L}(S) .
$$

We omit the proof of the dual statement.

THEOREM 4. NL (and hence NU) is isomorphic with $\tilde{C}$. 
Proof. Let $S$ be any subset of $C$. Wie first note that $S^{\wedge}=\sum(S)^{0}$ and that $x \leq \sum(S)^{0}$ if and only if $x \leq \sum(S)^{*}$. Hence

$$
\bar{S}=E_{x}\left(x \in C, x \leq \sum(S)^{*}\right) .
$$

It follows that

$$
\sum(\bar{S})=\left(\sum(S)^{*}\right)_{*} \cdot
$$

This if $S=\bar{S}$, then $\sum(S) \in N L$.

iNe now define a mapping $\phi$ of $\tilde{C}$ onto $N L$ by

$$
\phi(S)=\sum(S) \text {. }
$$

$\phi$ is onto $N L$ because

$$
x \in N L \rightarrow x \in L \rightarrow x=\sum\left(x_{0}\right),
$$

and by (2),

$$
\phi\left(\bar{x}_{0}\right)=\sum\left(\bar{x}_{0}\right)=\left(x^{*}\right)_{*}=x .
$$

Finally if $S \subset T \in \tilde{C}$, then $\phi(S) \leq \phi(T)$, while by (1),

$$
\phi(S) \leq \phi(T) \longrightarrow S=E_{x}\left(x \in C, x \leq \phi(S)^{*}\right) \subset E_{x}\left(x \in C, x \leq \phi(T)^{*}\right)=T .
$$

Using Theorem 4, we can show that $C$ may be characterized as follows: Let $A$ be a complete lattice which contains a subset $D$ isomorphic with $C$ and such that, for any $S \subset D$, whenever $\sum_{D}(S)$ or $\Pi_{D}(S)$ exists then it is equal to $\sum_{A}(S)$ or $\prod_{A}(S)$. If no proper subset of $A$ which contains $D$ is a complete lattice, then $A$ is isomorphic with $\tilde{C}$.

As applications of Theorem 4 we have the following examples. If $C$ is the class of open and closed subsets of a zero-dimensional space $Z$, then $\tilde{C}$ is isomorphic with the lattice of regular open sets of $Z$. For it is easily seen that if $x$ is any subset of $Z$, then

$$
x^{*}=\mathrm{C} x \text {, and } x_{*}=d x .
$$

If $C$ is a class of subsets of a set $A$ which contains all one-point subsets and their complements, then $\widetilde{C}$ is isomorphic with the lattice of all subsets of $A$. For $x=x_{*}=x^{*}$ whenever $x \subset A$. Our main application is given in the next section. 
A special result which we shall use later is the following.

THEOREM 5. If $B$ has the property that

$$
x \cap \sum_{x_{\alpha}}=\sum\left(x \cap x_{\alpha}\right)
$$

for any family $\left\{x_{\alpha}\right\}$, and

$$
\prod_{C}(S)=\prod(S)
$$

for any finite subset $S$ of $C$, then

$$
\prod_{L}(S)=\prod(S) \text { and } \prod_{N L}(S)=\prod(S)
$$

for any finite subset $S$ of $L$ and $N L$ respectively.

Proof. Let $x_{1}, \ldots, x_{n}$ be elements of $L$. Then

$$
x_{i}=\sum_{a \in I_{i}} y_{a, i} \text {, }
$$

where the $y_{\alpha, i}$ are elements of $\left(x_{i}\right)_{0}$. By $[1$, Theorem 14, p. 146], we have

$$
\prod_{x_{i}}=\sum_{f} \prod_{i} y_{f(i), i}
$$

where $f$ ranges over all functions such that $f(i) \in I_{i}$. Since $y_{f(i), i} \in C$, it follows that

$$
\prod_{x_{i}}=\sum_{f} y_{f},
$$

where $y_{f} \in C$. Hence $\prod_{x_{i}} \in L$, since $L$ is closed under $\sum$, and therefore

$$
\prod_{L}\left(x_{i}\right)=\prod_{x_{i}}
$$

If $x_{i} \in N L$, then

$$
\prod_{N L} x_{i}=\prod_{L} x_{i}=\prod_{x_{i}}
$$

2. The normal completion of lattices of continuous functions. Hereafter $B$ will stand for the set of all real-valued functions on a topologic space $X$. We allow the functions in $B$ to assume $\infty$ and $-\infty$ as values; $B$ is then a complete lattice in which

$$
\sum(S)(x)=\sup _{f \in S} f(x) .
$$


We define in $B$ the operations $f_{-}, f^{-}$, where

$$
\begin{aligned}
& f_{-}(x)=\sup _{A \in N(x)} \inf _{y \in A} f(y), \\
& f^{-}(x)=\inf _{A \in N(x)} \sup _{y \in A} f(y),
\end{aligned}
$$

$N(x)$ standing for the class of neighborhoods of $x$. It is well known that $f^{-}$and $f_{-}$are closure and dual closure operations on $B$. In accordance with Theorem 1 , define

$$
L S=L_{f}\left(f=f_{-}\right), U S=E_{f}\left(f=f^{-}\right)
$$

These are the classes of lower and upper semicontinuous functions on $X$. Ii also define the complete lattices

$$
N L S=E_{f}\left(f=\left(f^{-}\right)_{-}\right), \quad N \cup S=E_{f}\left(f=\left(f_{-}\right)^{-}\right)
$$

and call their members normal lower and upper semicontinuous. It is well known that $f \in L S \quad(f \in U S)$ if and only if $E(f>\lambda)(E(f<\lambda))$ is open for each finite $\lambda$. We now let $C=L S \cap U S$, the class of continuous, real-valued functions on $X$. If $A$. is a subset of $X$, then

$$
\left(k_{A}\right)^{-}=k_{C_{A}},\left(k_{A}\right)_{-}=k_{d_{A}}
$$

Hence $k_{A}$ is in $C, L S, U S, N L S$, or $N U S$ if and only if $A$ is open and closed, open, closed, regular open, or regular closed, respectively.

In terms of the present definitions of $B$ and $C$, let us now define the operations $f^{*}, f_{*}$ and the classes $L, U, N L$, and $N U$ as in $\S 1$. Theorem 4 specializes to:

THEOREM 6. The normal completion of the class of continuous, real-valued functions on an arbitrary topologic space $X$ is isomorphic with the class of $N L$ ( or NU) functions on $X$.

The lattice operations in the class $N L$ are described in Theorem 3 . It is easily seen that the hypotheses of Theorem 5 are satisfied in the present case. It follows that the greatest lower bound of a finite family of $L$ functions is also an $L$ function, and that the same is true of $N L$.

We now investigate the relations between the classes $L S, N L S, L$, and $N L$. We shall not state, but shall feel free to use, the duals of the next theorems. 
THEOREM 7. If $f \in B$, then $f_{*} \leq f_{-}$. Hence $L \subset L S$.

Proof. If $g \in C, g \leq f$, then $g=g_{-} \leq f_{-}$. Hence

$$
f_{*}=\sum\left(f_{0}\right) \leq f_{-} \text {. }
$$

COROLLARY. The relation

$$
\left(f_{*}\right)_{-}=\left(f_{-}\right)_{*}=f_{*}
$$

holds for every $f \in B$.

Proof. We have

$$
f_{*} \leq f_{-} \leq f \rightarrow f_{*}=\left(f_{*}\right)_{*} \leq\left(f_{-}\right)_{*} \leq f_{*}
$$

and applying the theorem to $f_{*}$, we have

$$
f_{*}=\left(f_{*}\right)_{*} \leq\left(f_{*}\right)_{-} \leq f_{*} .
$$

THEOREM 8. A necessary and sufficient condition that $f_{*}=f_{-}$for every $f \in B$ is that $X$ be completely regular.

Proof. Suppose $f_{*}=f_{-}$for every $f \in B$. Let $x$ be any point of $X$. Let $A$ be any neighborhood of $x$, and set $f=k_{A}$. Then $f \in L S$, and hence

$$
f=f_{-}=f_{*} \text {. }
$$

Therefore there is a continuous function $g$ with $g \leq f, g(x)>0$. This establishes the complete regularity of $X$.

Conversely, if $X$ is completely regular, suppose that for some $f \in B$ there is an $x$ with

$$
f_{*}(x)<f_{-}(x) .
$$

Then there is a neighborhood $A$ of $x$ with

$$
\inf _{y \in A} f(y)>\lambda>f_{*}(x) .
$$

Let $g \in C$ with $g(x)=1, g(y)=0$ for $y$ outside $A$, and $0 \leq g(z) \leq 1$ for all $z \in X$. Define

$$
h(y)=\lambda+\log g(y),
$$


with the convention that $\log 0=-\infty$. Then

$$
h \leq f, h \in C \text {, and } h(x)>f_{*}(x) .
$$

This contradiction completes the proof.

COROLLARY. If $X$ is completely regular then $\tilde{C} \cong N L S$.

We now obtain two characterizations of the class $N L S$.

LEMMA. For any $f \in B$ and any finite $\lambda$,

$$
E\left(f_{-} \geq \lambda\right)=n_{n} d E(f \geq \lambda-1 / n) .
$$

Proof. We have

$$
f_{-}(x) \geq \lambda \leftrightarrow \text { for every } n, x \in d E(f \geq \lambda-1 / n)
$$

THEOREM 9. If $f \in L S$, then $f \in N L S$ if and only if $E(f \geq \lambda)$ is an intersection of regular open sets for each finite $\lambda[2]$.

Proof. If $f \in N L S$, then

$$
E(f \geq \lambda)=E\left(\left(f^{-}\right)-\geq \lambda\right)=\cap_{n} d E\left(f^{-} \geq \lambda-1 / n\right),
$$

by the Lemma. The result now follows from the fact that $f^{-} \in U S$.

Conversely, if $f \in L S$, and $E(f \geq \lambda)$ is an intersection of regular open sets for each finite $\lambda$, we have

$$
f=f_{-} \leq\left(f^{-}\right)_{-} \cdot
$$

Suppose

$$
f(x)<\lambda<\left(f^{-}\right)_{-}(x)
$$

for some $x$. Then for some neighborhood $A$ of $x$ we have

$$
\inf _{y \in A} f^{-}(y)>\lambda
$$

But $E(f<\lambda)$ is a union of regular closed sets. Ilence there is an open set $B$ with $x \in C B$ and $f(y)<\lambda$ for each $y \in C B$. Thus $f^{-}(y) \leq \lambda$ for $y \in B$. But $B \cap A \neq 0$, since $x \in C B$. This contradiction completes the proof. 
THEOREM 10. If $f \in L S$, then $f \subset N L S$ if and only if $d E(f \geq \lambda)$ is a regular open set for each finite $\lambda$.

Proof. If $f \subset N L S$, then, by Theorem $8, E(f \geq \lambda)$ is an intersection of regular open sets, and hence its interior is regular open. Conversely, suppose $d E(f \geq \lambda)$ is regular open for each finite $\lambda$. We have

$$
E(f>\mu) \subset d E(f \geq \mu) \subset E(f \geq \mu)
$$

for each finite $\mu$. Hence

$$
E(f \geq \lambda)=\bigcap_{\mu<\lambda} E(f \geq \mu)=\bigcap_{\mu<\lambda} E(f>\mu)=\bigcap_{\mu<\lambda} d E(f \geq \mu),
$$

and the last is an intersection of regular open sets.

Other characterizations of $N L S$ are given in [2, Theorem 3.1], and in Theorem 14 below.

We now define $C_{1}$ as the class of finite real-valued continuous functions on $X$, and $C_{2}$ as the class of bounded real-valued continuous functions on $X$, and investigate the normal completions of these lattices. We shall use $\propto$ to denote the function which is identically $\infty$, and similarly for $-\propto$.

THEOREM 11. Let $M$ be the lattice consisting of all NLS functions which are bounded above and below by members of $C_{1}$ together with the functions $\infty,-\infty$. If $X$ is completely regular, then $\tilde{C}_{1} \cong M$.

Proof. We shall say that a function is dominated above or below if it is bounded above or below by a member of $C_{1}$. Let $f^{0}, f^{*}$ be defined as in $\xi 1$, where $C_{1}$ is used instead of $C$. We first note that if $f$ is dominated above, then $f^{*}=f^{-}$and both are dominated above. Indeed, if $f \leq h \in C_{1}$, then

$$
\begin{aligned}
f^{*}=\prod_{g \geq f, g \in C_{1}} g & =\prod_{h \geq g \geq f, g \in C_{1}} g \\
& =\prod_{h \geq g \geq f, g \in C} g=\prod_{g \geq f, g \in C} g=f^{-},
\end{aligned}
$$

by the dual of Theorem 8 . On the other hand, if $f^{0}=0$, then $f^{*}=\infty$.

Now the normal completion of $C_{1}$ is isomorphic with

$$
N L_{1}=E_{f}\left(f=\left(f^{*}\right)_{*}\right) \text {. }
$$

If $f$ is a finite-valued member of $M$, then $f^{*}=f^{-}$is dominated above and below. 
Hence

$$
\left(f^{*}\right)_{*}=\left(f^{*}\right)_{*}=\left(f^{-}\right)_{-}=f,
$$

and $f \in N L_{1}$. Conversely, if $f \in N L_{1}$ and $f \neq \infty, f \neq-\infty$, then $f$ must be dominated above. For otherwise $f^{*}=\infty$, and therefore

$$
\left(f^{*}\right)_{*}=f=\infty \text {. }
$$

Hence $f^{*}=f^{-}$, and $f^{*}$ is dominated above. Also $f^{-}$is dominated below, for otherwise

$$
f=\left(f^{-}\right)_{*}=-\propto
$$

Therefore

$$
f=\left(f^{*}\right)_{*}=\left(f^{-}\right)_{*}=\left(f^{-}\right)_{-},
$$

and $f \in M$.

In a similar manner we can prove:

THEOREM 12. If $X$ is completely regular, then $\tilde{C}_{2}$ is isomorphic with the lattice consisting of all bounded NLS functions together with the functions $\propto,-\infty[2]$.

We now prove a theorem of Stone [5] and Nakano [4].

THEOREM 13. If $X$ is extremally disconnected, then $C$ is complete. If $C$ is complete and $X$ is completely regular, then $X$ is extremally disconnected.

Proof. Suppose $X$ is extremally disconnected. Then by the lemma preceeding Theorem 9,

$$
f \in U S \rightarrow f_{-} \in U S \rightarrow f_{-} \in C .
$$

For any $f \in B$, we have $f^{*} \in U \subset U S$. Therefore $\left(f^{*}\right) \_\in C$. Consequently, for any $f$ in $N L$,

$$
f=\left(f^{*}\right)_{*}=\left(\left(f^{*}\right)_{-}\right)_{*}=\left(f^{*}\right)_{-},
$$

by the corollary of Theorem 9. Thus $N L=C$, and $C$ is complete.

Conversely, let $A$ be any regular open set. Then by Theorem 8 , 


$$
k_{A} \in N L S=N L=C \text {. }
$$

Therefore $A$ is closed.

3. Regular open sets and $N L S$ functions. Let $R(X)$, or simply $R$, denote the lattice of regular open sets of $X$. According to Theorem $3, R$ is a complete lattice in which

$$
\prod_{R}(S)=d \cap(S), \quad \sum_{R}(S)=d C U(S) \text {. }
$$

It is well known that $R$. is in fact a complete Boolean algebra. By Stone's Theorem, $R$ is isomorphic with the open and closed sets of an extremally disconnected, zero-dimensional, compact Hausdorff space, which we shall call the representation space of $R$.

THEOREM 14. A necessary and sufficient condition that $f$ be normal lower semicontinuous is the existence of a family $\left\{x_{\lambda}\right\},-\propto<\lambda<\propto$, of regular open sets with the properties

i) $x_{\lambda} \subset x_{\mu}$ for $\lambda>\mu$,

ii) $f(p)=\sup E_{\lambda}\left(p \in x_{\lambda}\right)$.

In this case we have

iii) $d E(f \geq \lambda)=\prod_{R, \mu<\lambda} x_{\mu}$.

If $f \in N L S$, we may choose $x_{\lambda}=d E(f \geq \lambda)$.

Proof. Suppose $f \in N L S$. I et

$$
x_{\lambda}=d E(f \geq \lambda) .
$$

Theorem 10 shows that $x_{\lambda} \in R$, and the proof of that theorem shows that iii) holds. Since i) is obvious, it remains to prove ii). Suppose $\lambda<f(p)$. Then

$$
p \in E(f>\lambda) \subset x_{\lambda}
$$

Hence

$$
\sup E_{\lambda}\left(p \in x_{A}\right) \geq f(p) \text {. }
$$

On the other hand, if $\lambda>f(p)$, then 


$$
p \not \subset E(f \geq \lambda) \supset x_{\lambda} \text {. }
$$

Therefore

$$
\sup E_{\lambda}\left(p \in x_{\lambda}\right) \leq f(p) .
$$

Now suppose $\left\{x_{\lambda}\right\}$ is a family of gular open sets satisfying i), and let $f$ be defined by ii). If $f(p)>\lambda$, then $p \in x_{\mu}$ for some $\mu>\lambda$. But

$$
f(q) \geq \mu>\lambda \quad \text { for } q \in x_{\mu} .
$$

Therefore $E(f>\lambda)$ is open and $f \in L S$. Also

$$
E(f \geq \lambda)=\cap_{\mu<\lambda} x_{\mu},
$$

which implies iii). Theorem 10 now shows that $f \in N L S$.

THEOREM 15. If $X$ and $Y$ are any topologic spaces, then $N L S(X) \cong N L S(Y)$ if and only if $R(X) \cong R(Y)$. In particular ([2] when $X$ is completely regular), if $Z$ is the representation space of $R(X)$, then $N L S(X) \cong C(Z)$.

Proof. Suppose $R(X) \cong R(Y)$. Let $\phi$ be a function on $R(X)$ to $R(Y)$ which establishes this isomorphism. If $f \in N L S(X)$, let

$$
x_{\lambda}=d E(f \geq \lambda),
$$

Define $T f$ to be the function on $Y$ defined by

$$
T f(p)=\sup E_{\lambda}\left(p \in \phi\left(x_{\lambda}\right)\right) .
$$

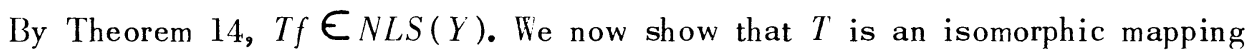
of $N L S(X)$ onto $N L S(Y)$. If $f, g \in N L S(X)$, let

$$
x_{\lambda}=d E(f \geq \lambda), y_{\lambda}=d E(g \geq \lambda) .
$$

Clearly,

$$
f \geq g \rightarrow x_{\lambda} \supset y_{\lambda} \rightarrow \phi\left(x_{\lambda}\right) \supset \phi\left(y_{\lambda}\right)
$$

for every $\lambda$, and therefore $T f \geq T g$. Using Theorem 14, iii ) twice, we have

$$
x_{\lambda}=\prod_{R(X), \mu<\lambda} x_{\mu},
$$

which implies 


$$
\dot{\phi}\left(x_{\lambda}\right)=\prod_{R(Y), \mu<\lambda} \phi\left(x_{\mu}\right)=d E(T f \geq \lambda) .
$$

From this it follows that

$$
T f \geq T g \longrightarrow d E(T f \geq \lambda) \supset d E(T g \geq \lambda) \longrightarrow \phi\left(x_{\lambda}\right) \supset \phi\left(y_{\lambda}\right) \longrightarrow x_{\lambda} \supset y_{\lambda}
$$

for every $\lambda$. This implies $f \geq g$ by Theorem 14, ii). Finally, if $F \in N L S(Y)$, choose $x_{\lambda} \in R(X)$ so that

$$
\phi\left(x_{\lambda}\right)=d E(F \geq \lambda),
$$

and define $f$ as in Theorem 14, ii $)$. Then $f \in N L S(X)$ and

$$
d E(f \geq \lambda)=\prod_{R(X), \mu<\lambda x_{\mu}},
$$

and this last is $x_{\lambda}$ because

$$
\phi\left(x_{\lambda}\right)=\prod_{R(Y), \mu<\lambda} \phi\left(x_{\mu}\right) .
$$

This shows that $T f=F$.

If $Z$ is the representation space of $R(X)$, then $N L S(Z)=C(Z)$ by Theorem 13 and the corollary to Theorem 8.

Now suppose $N L S(X) \cong N L S(Y)$. Let $Z_{1}$ and $Z_{2}$ be the representation spaces of $R(X)$ and $R(Y)$, respectively. Then

$$
C\left(Z_{1}\right) \cong C\left(Z_{2}\right) \text {. }
$$

A theorem of Kaplansky [3] shows that $Z_{1}$ is homeomorphic with $Z_{2}$. This implies that

$$
R(X) \cong R(Y),
$$

and the proof is complete.

In Theorem 14, it is easily seen that $f$ is bounded if and only if $x_{\lambda}=0$ for all $\lambda$ outside some finite interval. It follows from the proof of Theorem 15 that the bounded NLS functions on $X$ are isomorphic with those on $Y$ if and only if

$$
N L S(X) \cong N L S(Y) \text {. }
$$

The same is not true of the finite valued $N L S$ functions. If

$$
R(X) \cong R(Y),
$$


all we can say is that the classes of $N L S$ functions $\int$ on $X$ and $Y$, respectively, which are such that $E(|f|=\propto)$ is nowhere dense, are isomorphic. This follows from the fact that $E(f=\infty)$ is nowhere dense if and only if

$$
\prod_{R} x_{\lambda}=0
$$

and $E(f=-\infty)$ is nowhere dense if and only if

$$
\sum_{R} x_{\lambda}=X \text { or } Y \text {. }
$$

The following theorem gives a corresponding result for the lattices of continuous functions. We recall that $C_{2}(X)$ denotes the lattice of bounded realvalued continuous functions on $X$.

THEOREM 16. If $X$ and $Y$ are any topologic spaces, then

$$
C_{2}(X) \cong C_{2}(Y)
$$

if and only if

$$
C(X) \cong C(Y) \text {. }
$$

Proof. There exist completely regular Hausdorff spaces $X^{\prime}, Y^{\prime}$ such that

$$
C_{2}(X) \cong C_{2}\left(X^{\prime}\right), C_{2}(Y) \cong C_{2}\left(Y^{\prime}\right), C(X) \cong C\left(X^{\prime}\right) \text {, and } C(Y) \cong C\left(Y^{\prime}\right)
$$

( one modifies Dilworth's proof $[2, \xi 2]$ by defining $x \sim y$ if $f(x)=f(y)$ for all $f \in C(X))$. Now let $\beta X^{\prime}, \beta Y^{\prime}$ be the Stone-Čech compactifications of $X^{\prime}, Y^{\prime}$. Then

$$
C_{2}\left(\beta X^{\prime}\right) \cong C_{2}\left(X^{\prime}\right)
$$

Now the mapping

$$
\phi(f)=f /(1+|f|)
$$

carrier $C\left(X^{\prime}\right)$ into $C_{2}\left(X^{\prime}\right)$. We use this to extend members of $C\left(X^{\prime}\right)$. Thus

$$
C\left(\beta X^{\prime}\right) \cong C\left(X^{\prime}\right) .
$$

Combining these facts, we have

$$
\begin{aligned}
C(X) \cong C(Y) \leftrightarrow C\left(X^{\prime}\right) & \cong C\left(Y^{\prime}\right) \leftrightarrow C\left(\beta X^{\prime}\right) \cong C\left(\beta Y^{\prime}\right) \\
\leftrightarrow & \beta X^{\prime} \text { is homeomorphic with } \beta Y^{\prime}
\end{aligned}
$$


(by Kaplansky's Theorem [3]), ↔

$C_{2}\left(\beta X^{\circ}\right) \cong C_{2}\left(\beta Y^{\prime}\right)$ (again by $\left.[3]\right) \leftrightarrow C_{2}\left(X^{\prime}\right) \cong C_{2}\left(Y^{\prime}\right) \leftrightarrow C_{2}(X) \cong C_{2}(Y)$.

It would be of interest to give a more elementary proof of this theorem.

\section{REFERENCES}

1. G. Birkhoff, Lattice theory, Amer. Math. Soc. Colloquium Publications, vol. XXV, Revised Edition, New York, 1948.

2. R. P. Dilworth, The normal completion of the lattice of continuous functions, Trans. Amer. Math. Soc. 68 (1950), 427-438.

3. I. Kaplansky, Lattices of continuous functions, Bull. Amer. Math. Soc. 53 (1947), $617-622$.

4. H. Nakano, Über das System aller stetigen Funktionen auf einem topologischen Raum, Proc. Imp. Acad. Tokyo 17 (1941), 308-310.

5. M. H. Stone, Boundedness properties in function lattices, Canadian J. Math. 1 (1949), $176-186$.

University of California, Los Angeles, California 


\section{PACIFIC JOURNAL OF MATHEMATICS}

\section{EDITORS}

\author{
R. M. Foeinson \\ University of California \\ Berkeley 4, California \\ E. HEWITt \\ University of Washington \\ Seattle 5, Washington
}

R. P. DILWOR TH

California Institute of Technology

Pasadena 4, California

E. F. BECKENBACH

University of California

Los Angeles 24, California

\section{ASSOCIATE EDITORS}

$\begin{array}{llll}\text { H. BUSEMANN } & \text { P. R. HALMOS } & \text { BøRGE JESSEN } & \text { J. J. STOKER } \\ \text { HERBERT FFDERER } & \text { HEINZ HOPF } & \text { PAUL LÉVY } & \text { E. G. STR AUS } \\ \text { MARSHALL IIALI } & \text { R. D. JAMES } & \text { GEORGE PÓLYA } & \text { KỎSAKU YOSIDA }\end{array}$

\section{SPONSORS}

UNIVERSITY OF BRITISH COLUMBIA CAIIFORNIA INSTITUTE OF TECHNOLOGY UNIVERSITY OF CALIFORNIA, BERKELEY UNIVERSITY OF CAILIFORNIA, DAVIS UNIVERSITY OF CALIFORNIA, LOS ANGELES UNIVERSITY OF CALIFORNIA, SANTA BARBARA UNIVERSITY OF NEVADA OREGON STATE COLLEGE UNIVERSITY OF OREGON
UNIVERSITY OF SOUTHERN CALIFORNIA STANFORD RESEARCH INSTITUTE STANFORD UNIVERSITY WASHINGTON STATE COLLEGE UNIVERSITY OF WASHINGTON

AMERICAN MATHEMATICAL SOCIETY NATIONAL BUREAU OF STANDARDS, INSTITUTE FOR NUMERICAL ANALYSIS

$$
\begin{gathered}
\text { Vari-Type Composition by } \\
\text { Elaine Barth } \\
\text { Delores Wierman } \\
\text { With the cooperation of } \\
\text { E. F. Beckenbach } \\
\text { E. G. Straus }
\end{gathered}
$$

Printed in the United States of America by Edwards Brothers, Inc., Ann Arbor, Michigan 


\section{Pacific Journal of Mathematics}

\section{Vol. 3, No. 1 \\ March, 1953}

Herbert Busemann, Volume in terms of concurrent cross-sections ......... 1

L. Carlitz, Some special equations in a finite field ................. 13

Homer V. Craig and Billie Braden Townsend, On certain metric

extensors ....................................... 25

Philip J. Davis and Henry Pollak, Linear functionals and analytic

continuation problems ............................. 47

Jacob C. E. Dekker, The constructivity of maximal dual ideals in certain

Boolean algebras ................................. 73

Harley M. Flanders, The norm function of an algebraic field extension .... 103

Marshall Hall, Subgroups of free products . . . . . . . . . . . . . . . . . 115

Israel (Yitzchak) Nathan Herstein, Finite multiplicative subgroups in

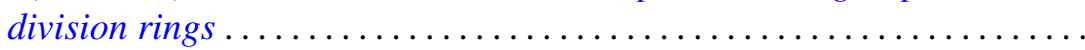

Joseph Lawson Hodges, Jr. and Murray Rosenblatt, Recurrence-time moments in random walks ............................ 127

Alfred Horn, The normal completion of a subset of a complete lattice and lattices of continuous functions ........................ 137

Fulton Koehler, Estimates for the errors in the Rayleigh-Ritz method...... 153

M. H. Martin, The Monge-Ampère partial differential equation

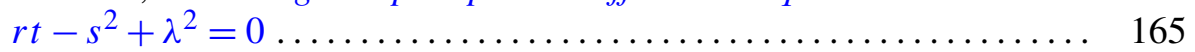

John E. Maxfield, Normal k-tuples ......................... 189

Jack E. McLaughlin, Structured theorems for relatively complemented lattices............................................ 197

William H. Mills, A system of quadratic Diophantine equations ......... 209

T. S. Motzkin, Ernst Gabor Straus and F. A. Valentine, The number of farthest points ................................. 221

G. Power, Forces on the boundary of a dielectric ............... 233

Ralph Gordon Selfridge, Approximations with least maximum error....... 247 